\title{
Azathioprine Sodium
}

National Cancer Institute

\section{Source}

National Cancer Institute. Azathioprine Sodium. NCI Thesaurus. Code C47961.

The sodium salt form of azathioprine, a pro-drug of purine analogue with

immunosuppressive activity. Azathioprine is converted in vivo to its active metabolite 6-

mercaptopurine (6-MP), which substitutes for the normal nucleoside and mistakenly gets

incorporated into DNA sequences. This leads to inhibition of DNA, RNA, and protein

synthesis. As a result, cell proliferation may be inhibited, particularly in lymphocytes and leukocytes. 